\title{
Una experiencia de innovación docente aplicada a la asignatura Metodología Arqueológica
}

\section{An experience of teaching innovation applied to the subject Archaeological Methodology}

DIEGO ROMERO VERA

ORCID: https://orcid.org/0000-0002-4562-2407

Universidad de Sevilla. Departamento

de Prehistoria y Arqueología

drvera@us.es

Fecha de recepción: 02-06-2019

Fecha de aceptación: 16-06-2019

DOI: http://dx.doi.org/10.12795/9788447221912.020 Pp.: 469-493 


\section{Resumen}

En este trabajo presentamos el diseño y desarrollo del Ciclo de Mejora en el Aula implementado en la asignatura Metodología arqueológica, en el Doble Grado en Geografía y Gestión del Territorio e Historia durante el curso académico 2018/2019. Dicho ciclo de mejora se basa en la aplicación del modelo metodológico de construcción del conocimiento y ha arrojado un resultado bastante positivo, palpable en la evolución del aprendizaje de los alumnos.

Palabras claves: Metodología arqueológica; Doble Grado en Geografía y Gestión del Territorio e Historia; Docencia universitaria; Experimentación docente universitaria.

\section{Abstract}

In this work we introduce the design and development of an Improvement Cycle in Classroom applied to the subject Archaeological Methodology, in the Dual Degree in Geography, Land Management and History during the academic year 2018/2019. This improvement cycle is based on the implementation of methodological model known as knowledge building, and it has produced a quite positive result evident in the evolution of student's learning.

Keywords: Archaeological Methodology; Dual Degree in Geography, Land Management and History; University teaching; University teaching experimentation.

Jornadas de Formación e Innovación Docente del Profesorado | № 2 (2019) Esta obra se distribuye con la licencia Creative Commons 


\section{Breve descripción del contexto}

Este ciclo de mejora en el aula (CIMA) se aplica en la asignatura Metodología Arqueológica, del Doble Grado en Geografía y Gestión del Territorio e Historia (Plan 2010). La asignatura se imparte en el segundo cuatrimestre del primer curso del grado y se trata de una asignatura troncal. Esta se plantea como introducción general a la arqueología como disciplina histórica y como método de trabajo, atendiendo, por una parte, a su contenido: enfoques teóricos, campos de aplicación que se traducen en formas diferentes de especialización, y, por otra, a las técnicas más usuales de trabajo de campo y laboratorio.

La asignatura en cuestión es ardua. Por un parte, aborda los diferentes enfoques epistemológicos que se han ofrecido de la arqueología desde su desarrollo como disciplina científica. Se trata de un asunto de relativa complejidad que requiere que el alumnado posea ciertos conocimientos previos relacionados con la filosofía de la ciencia. Por otra parte, la asignatura acomete contenidos relativos a la aplicación de técnicas de investigación arqueológica con métodos e instrumental técnico. Además, en la materia abundan los conceptos abstractos. No hay que perder de vista que es una asignatura troncal de primer curso, es decir, es el primer contacto que tienen los alumnos con la disciplina arqueológica.

La metodología de enseñanza-aprendizaje se ha basado en la típica clase expositiva, esto es, lecciones tradicionales desarrolladas en clase en la que el profesor aborda explicaciones sobre los contenidos de la materia. Normalmente, la clase teórica está abierta al diálogo con los alumnos, con bibliografía orientativa y empleo de técnicas audiovisuales informatizadas. Las lecciones se llevan a cabo los lunes y martes de 12 a 14 horas con un breve descanso a las 13 horas. El ciclo de mejora se ha desarrollado durante 6 horas. 


\section{Diseño previo del Ciclo de Mejora en el Aula}

\section{Modelo metodológico}

Frente al modelo metodológico que he desarrollado hasta ahora, la denominada clase expositiva, en este ciclo de mejora vamos a implementar el aprendizaje basado en la resolución de problemas (Morales y Landa, 2004; Prieto, 2006). Esta metodología persigue que los estudiantes solventen problemas de la vida real, en nuestro caso concreto, las cuestiones que debe plantearse un arqueólogo o investigador de esta rama del saber en el desempeño de su labor cotidiana, mediante el conocimiento, la investigación y la reflexión. En concreto, se propone a los alumnos un problema o cuestión que ellos deben solventar a través de la búsqueda y el tratamiento de información. Dicha metodología se sintetiza en el siguiente esquema (Figura 1).

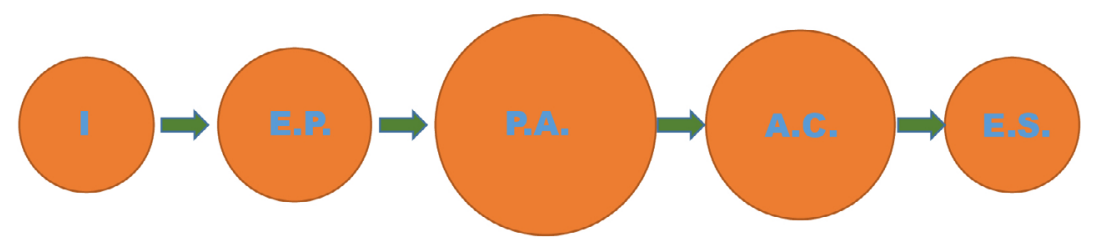

I.: Introducción; E.P.: Exposición del problema; P.A.: Propuestas de los alumnos; A.C.: Actividades de contraste; E.S.: Elaboración de una síntesis

Figura 1: Esquema del desarrollo de la metodología.

\section{Mapa de contenidos}

El mapa de contenidos (Figura 2) recoge la materia a impartir sobre el tema al que se consagra este ciclo de mejora. Los dos conceptos principales que giran en torno al yacimiento arqueológico se ofrecen a modo de pregunta: ¿qué es? (en color verde) y ¿cómo se forma? (en color amarillo). Así pues, por una parte, se detalla qué es un yacimiento arqueológico, se desglosa su definición, se 
describen sus características y la división tipológica. Por lo que respecta a su formación, se describen las diversas fases que dan lugar a la creación de un yacimiento y los procesos postdeposicionales que los yacimientos experimentan, sin olvidar las técnicas que permiten estudiar la formación de éstos. Dentro de una cartela de color rojo, y unidos a los conceptos por flechas, se desgranan los procedimientos y competencias que deben desarrollar los alumnos en el marco de este tema. Por lo que respecta a la definición, deben elegir la más completa y precisa de entre las muchas que los especialistas en la materia han ofrecido. Asimismo, deben tener presente la importancia que los vestigios materiales presentan en la ciencia arqueológica; de hecho, este aspecto es un pilar fundamental a la hora de definir qué es un yacimiento.

Jornadas de Formación e Innovación Docente del Profesorado | № 2 (2019) Esta obra se distribuye con la licencia Creative Commons 


\section{DIEGO ROMERO VERA}

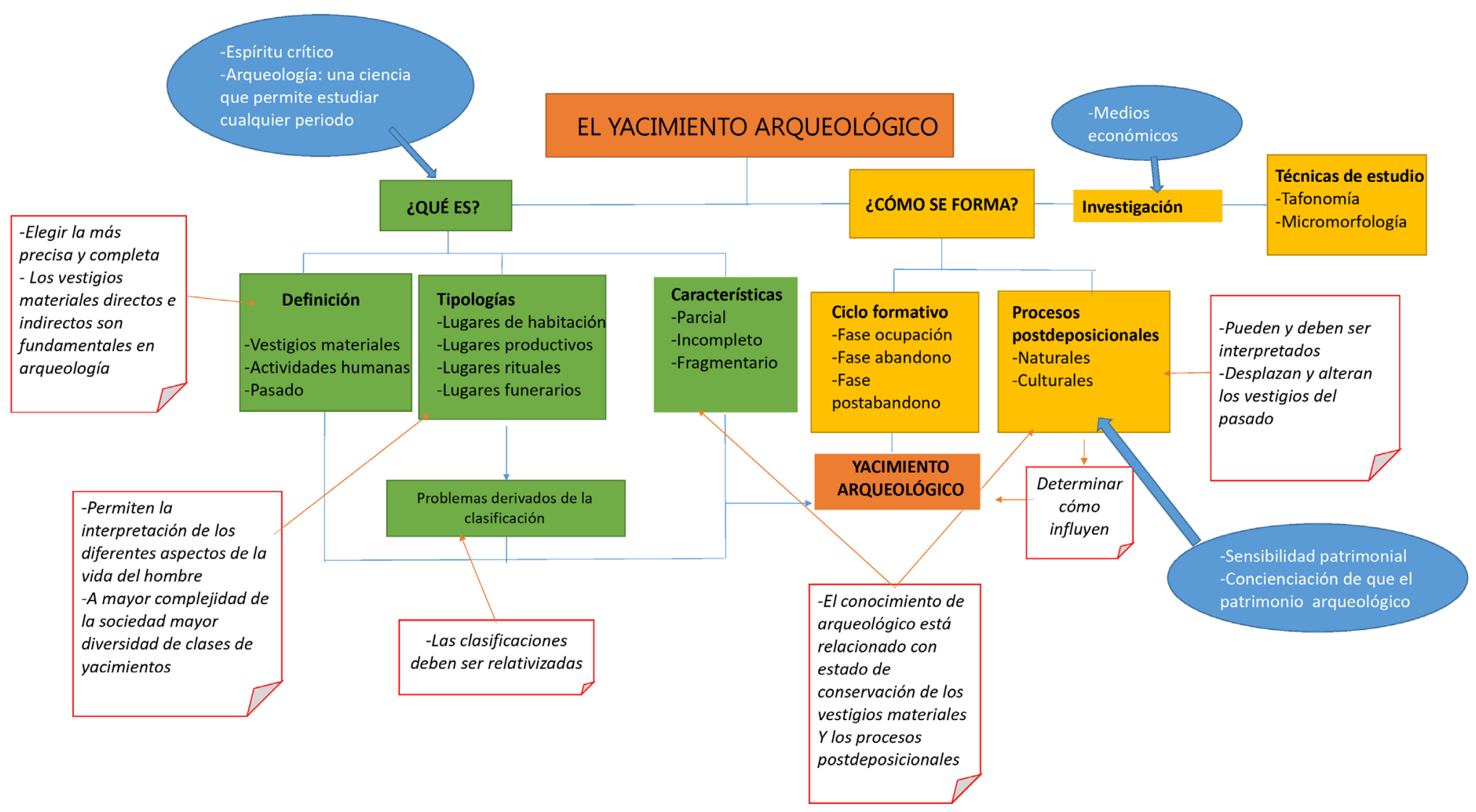

Figura 2: Mapa de contenidos.

Jornadas de Formación e Innovación Docente del Profesorado | № 2 (2019)

cc (7) $\odot$ Esta obra se distribuye con la licencia Creative Commons 
En cuanto a las tipologías de yacimientos, constituyen una clave para interpretar diversos aspectos de la vida humana. En este sentido, los alumnos deben conocer en qué consisten y cuáles son los procesos postdeposicionales, y también cómo afectan a los yacimientos estos fenómenos que deben ser analizados por el arqueólogo. En relación a esto último, los alumnos deben comprender que el conocimiento arqueológico está relacionado con el estado de conservación de los vestigios materiales y los procesos postdeposicionales. Ningún mapa de contenidos estaría completo sin incluir los contenidos actitudinales (en color azul). A la hora de definir qué es un yacimiento arqueológico debe aflorar un cierto espíritu crítico para elegir una definición de referencia; asimismo los alumnos deben saber que la arqueología es un método que permite estudiar cualquier periodo histórico y no solo la prehistoria o el mundo clásico. Un proceso postdeposicional muy perjudicial es la búsqueda ilícita de antigüedades; al respecto, es necesario poseer cierta sensibilidad y tomar conciencia de que el patrimonio arqueológico es único y finito. Finalmente, hay que entender que toda actividad arqueológica implica un desembolso económico y que hay que establecer una serie de prioridades y objetivos antes de iniciar cualquier proceso de investigación.

\section{Secuencia de actividades programada}

La secuencia de actividades a aplicar se divide en cuatro partes, como se ha indicado, primando el aprendizaje activo del alumnado que el profesor deberá guiar (véase Figura 3). Se aplica al tema "El yacimiento arqueológico, unidad base del trabajo de campo"; a estos contenidos se destinan 6 horas que corresponden, a su vez, a 3 clases, ya que cada una de ellas tiene una duración de 2 horas.

Jornadas de Formación e Innovación Docente del Profesorado | № 2 (2019) Esta obra se distribuye con la licencia Creative Commons 


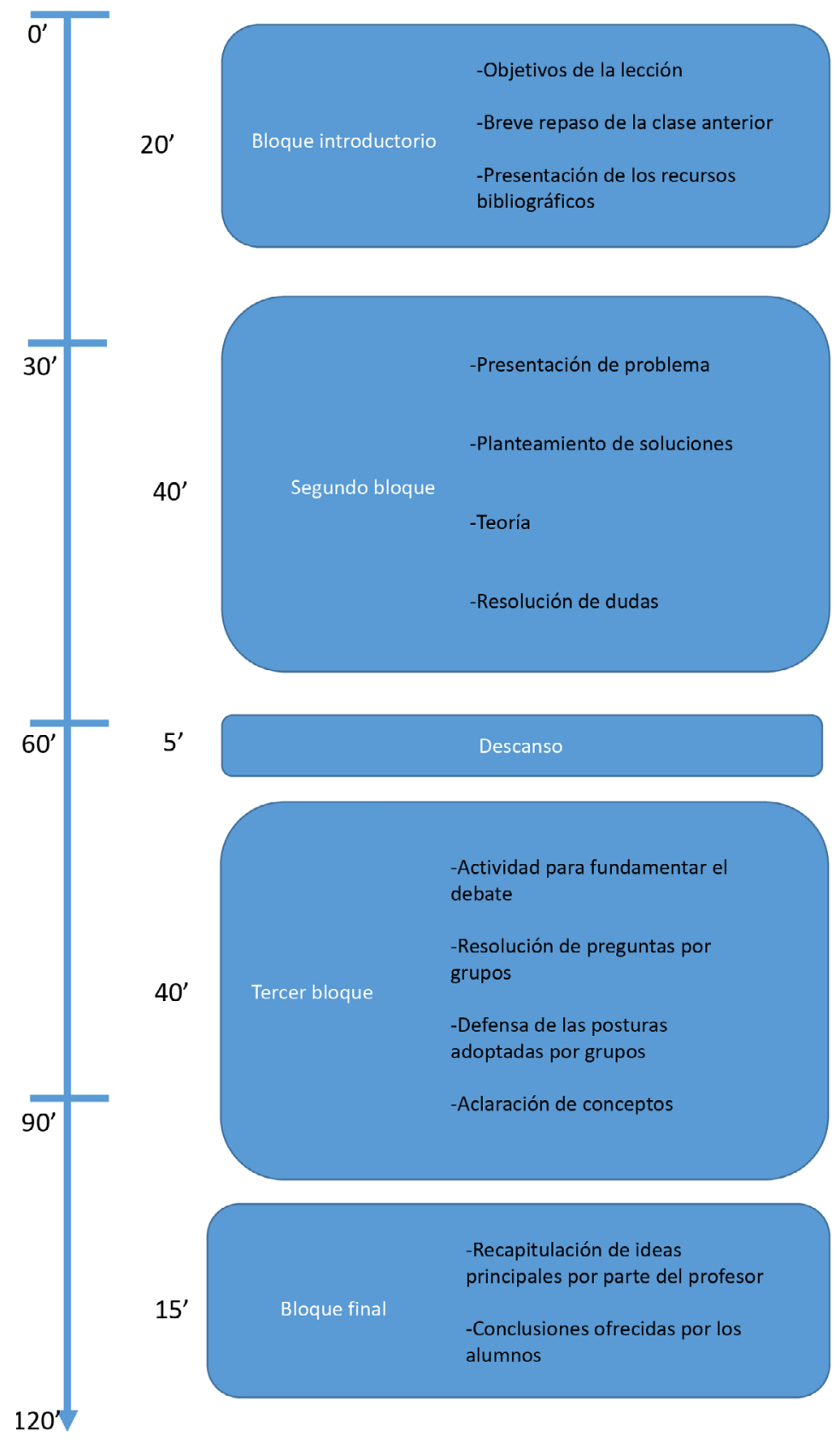

Figura 3: Esquema-sintesis del desarrollo de las actividades en una sesión de 2 horas de clase.

Jornadas de Formación e Innovación Docente del Profesorado I № 2 (2019) Esta obra se distribuye con la licencia Creative Commons Reconocimiento-NoComercial-SinObraDerivada $\quad 4.0$ Internacional (CC BY-NC-ND 4.0.) 
La clase comienza con una breve exposición de las metas de aprendizaje y objetivos formativos de la lección. Esta actividad sirve para contextualizar qué van a aprender y a través de qué procedimientos. Asimismo, se enlaza esta lección con los contenidos vistos en clases anteriores, de forma que el alumno perciba que existe una sucesión en la materia de la asignatura. A continuación, el profesor ofrece bibliografia sobre los aspectos que se van a tratar en la clase del día. Él es el encargado de presentar esas obras (que pueden ser manuales, artículos de investigación o monografías). Posteriormente, esa bibliografía irá rotando entre los alumnos para que durante la clase puedan consultar la obra, leer la introducción, analizar el indice y, sobre todo, anotar estas referencias para que más tarde puedan acceder a ellas de cara a ampliar el tema.

El segundo bloque se inicia con el planteamiento de un problema que servirá de pórtico a la introducción de contenidos. Con este fin, se divide la clase en grupos y el profesor va guiando la resolución de este ejercicio. Este problema se irá complejizado paulatinamente; para ello el docente propicia que los alumnos ofrezcan soluciones creativas a los problemas ofrecidos (Finkel, 2008). En cualquier caso, es básico que este desarrolle un refuerzo positivo y evite la sensación de miedo a equivocarse, ya que es fundamental que aprendan de los errores. Este apartado finaliza con el esclarecimiento del problema inicial. Acto seguido, se desarrolla una breve explicación de contenidos teóricos y aclaración de conceptos. En este apartado el protagonismo vuelve momentáneamente al docente. No obstante, existe una reciprocidad con sus alumnos, pues precisamente el docente debe favorecer que ellos lancen preguntas y aclaren las dudas que la explicación de conceptos teóricos ha generado.

La tercera parte comienza con el desempeño de una actividad que sirve de fundamento para el desarrollo de un debate posterior. Me estoy refiriendo a la lectura de 
un extracto de una obra científica o noticia periodística, el visionado de un breve vídeo o la exposición de un caso práctico real o ficticio. A continuación, el profesor ofrece un listado de preguntas ciertamente complejas relacionadas con la actividad previa; cada grupo dispone de un breve periodo de tiempo para ofrecer respuestas circunstanciadas, que previamente deben ser acordadas entre todos sus miembros. Esto induce a los alumnos a reflexionar sobre cuál debe ser la respuesta correcta y, por ende, a aplicar de forma razonada lo que han aprendido. Cuando todos han ofrecido su punto de vista, se genera un debate entre los grupos en el que deben defender el posicionamiento que les ha llevado a dar esa solución y no otra. En las réplicas los estudiantes deben evaluar las opciones que se presentan y desarrollar un pensamiento crítico. En todo momento, el docente debe servir de guía y estimular el debate. En última instancia, se persigue que los alumnos sepan definir y aislar el problema ofrecido, tasar la credibilidad de las fuentes, reconocer la endeblez de ciertos argumentos y ofrecer posicionamientos basados en la materia de la lección. Además, los debates sirven para que los alumnos desarrollen habilidades comunicativas. Este apartado se cierra con la intervención del profesor para puntualizar los diferentes argumentos y aclarar los conceptos que han salido a la palestra en esta actividad.

Por último, el docente ofrece una recapitulación de las ideas principales y recuerda la importancia de ampliar conocimientos a partir de la bibliografía comentada en el inicio de clase. Asimismo, se insta a los alumnos a que expresen verbalmente cuáles son los conceptos esenciales que se han desgranado a lo largo de la clase.

Para realizar el CIMA se proyectaron una serie de actividades y, también, un cuestionario inicial (vid. infra) con el que valorar las ideas iniciales y finales de los alumnos sobre el yacimiento como unidad base del trabajo de campo (Rivero y Porlán, 2017). 
Actividad 1. Selección de una definición precisa de yacimiento arqueológico + Cuestionario inicial

- Descripción: Se emplaza a los alumnos a que definan por sus propios medios qué es un yacimiento arqueológico. A continuación, el profesor ofrece un texto con una batería de definiciones que han ofrecido diferentes especialistas en este campo de estudio sobre el concepto indicado. Finalmente, ellos deben elegir de forma consensuada cuál les parece la más precisa y completa.

- Sentido formativo: Con esta actividad el alumnado concibe la multiplicidad de puntos de vista diferentes que pueden existir acerca de lo que es un yacimiento. Cada autor ofrece una similar; ellos deben profundizar en los matices que encierra cada una de estas definiciones y asumir, por tanto, un pensamiento crítico que fundamente la selección de una que les sirva de referente.

Actividad 2. Base teórica sobre los elementos que componen el registro arqueológico

- Descripción: Explicación de los contenidos fundamentales que componen el registro arqueológico, es decir, unidades estratigráficas, artefactos y ecofactos, empleando para este fin una breve exposición oral apoyada en una presentación Powerpoint.

- Sentido formativo: Se imparten conceptos básicos sobre el tema referido. En la asignatura existe otro tema que se consagra a analizar en profundidad el concepto de unidad estratigráfica y la clasificación del registro arqueológico. No obstante, en este tema se alude a estos aspectos fundamentales que es necesario aclarar a través de una breve explicación teórica.

Actividad 3. Debate y puesta en común de posturas acerca de las características fundamentales del registro arqueológico 
- Descripción: Se ofrece a los alumnos dos imágenes, una de ellas representa a cazadores-recolectores en una representación que recrea una escena de vida cotidiana, se insta a los alumnos a que se fijen en su atuendo, elementos de adorno, utillaje y armamento. En la otra imagen aparecen los materiales, casi todos ellos líticos, fruto de la excavación de un hábitat de época paleolítica. A continuación, el profesor ofrece tres preguntas: ¿el registro arqueológico nos permite recrear con total fiabilidad la vida en la prehistoria?, ¿qué ocurre con los vestigios que tienen un origen orgánico?, ¿la tecnología del Paleolítico se basaba exclusivamente en la piedra? Cada uno de los grupos debe elegir por consenso cuáles son las soluciones a los interrogantes propuestos. A continuación, se genera un debate entre los representantes de los grupos, cada uno de ellos debe argumentar su respuesta y elegir, de nuevo por consenso, cuál es la repuesta más fundamentada. La actividad finaliza con la aclaración de conceptos por parte del profesor.

- Sentido formativo: Con esta actividad se persigue que los alumnos capten que el registro arqueológico es parcial, fragmentario e incompleto. La actividad les lleva a reflexionar sobre la cantidad de artefactos de origen orgánico - pieles, enmangues de armas, cestas, etc.- que emplearon los hombres y mujeres del Paleolítico. Debido a que han desaparecido, el arqueólogo corre el riesgo de recrear una imagen muy sesgada de la cultura material y de la tecnología de las sociedades del pasado.

Actividad 4. Aprendiendo a distinguir entre registro arqueológico y yacimiento arqueológico

- Descripción: Se define por parte del profesor la relación que existe entre yacimiento y registro arqueológico. A continuación, se ofrece a los alumnos dos textos periodísticos que hacen alusión al hallazgo de 
una inscripción romana, por un lado, y de un tesoro de monedas medievales, por otro. En ambos casos, la noticia relata que fueron hallados en mitad de parajes aislados, sin vinculación con un yacimiento arqueológico conocido o hábitat determinado. Se plantea a los alumnos el siguiente problema: Teniendo en cuenta la definición de yacimiento vista en la clase anterior ¿estos dos elementos arqueológicos citados deberían categorizarse como yacimientos?

- Sentido formativo: La actividad incide en la dificultad de categorizar conceptos tales como registro y yacimiento arqueológico; la definición de yacimiento engloba al registro material, pero a veces encontramos situaciones anómalas que escapan a tales definiciones. En este caso, una inscripción o un tesoro no suponen por sí mismo yacimientos arqueológicos.

Actividad 5. Base teórica sobre los procesos que dan lugar a la formación del registro arqueológico

- Descripción: Explicación de los contenidos fundamentales relacionados con el ciclo de formación del registro arqueológico, empleando para este fin una breve exposición oral apoyada en una presentación Powerpoint.

- Sentido formativo: Se imparten conceptos básicos sobre el tema referido. Estos conceptos y procesos son complejos, por lo que es necesario que el profesor los explique brevemente en clase, haciendo hincapié en lo verdaderamente importante y dejando de lado cuestiones superfluas.

Actividad 6. Debate y puesta en común de posturas acerca de las características de los yacimientos funerarios

- Descripción: La actividad comienza con la proyección de tres breves vídeos sobre tres yacimientos funerarios de diferentes etapas históricas: pirámides egipcias del Valle de los Reyes, necrópolis romana de Isola Sacra en Ostia y la maqbara medieval de 
Ávila. A continuación, los alumnos, divididos en grupos, deben responder a tres preguntas: ¿cuáles son los rasgos principales de los lugares o yacimientos funerarios?, ¿en qué se diferencian de los lugares de habitación?, ¿por qué los artefactos recuperados en las tumbas suelen presentar un excelente nivel de conservación? Cada uno de los grupos debe elegir por consenso cuáles son las soluciones a los interrogantes propuestos. A continuación, se genera un debate entre los representes de los grupos; cada uno de ellos debe argumentar su respuesta y elegir, de nuevo por consenso, cuál es la repuesta más fundamentada. La actividad finaliza con la aclaración de conceptos por parte del profesor.

- Sentido formativo: Con esta actividad se persigue que los alumnos descubran a través de su propio razonamiento cuáles son las características más definitorias del registro arqueológico funerario, tanto desde el punto de vista deposicional como intepretativo.

Actividad 7. Alteración del registro arqueológico: expolio y reutilizaciones

- Descripción: En esta actividad se proyecta una fotografía de una plaza de Roma en la que se observa un obelisco egipcio que presenta una inscripción latina en su base y una cruz en su vértice superior. Con relación a la imagen y al tema que estamos tratando en clase, planteamos la siguiente pregunta: ¿es el expolio una actividad contemporánea? Los alumnos deben meditar su respuesta. A continuación, el profesor pide a varios estudiantes que ofrezcan su explicación al interrogante propuesto. Acto seguido, el profesor ofrece la solución, matizando las respuestas que ha recibido.

- Sentido formativo: Con esta actividad se busca que los estudiantes conozcan que la actividad expoliadora no es un fenómeno reciente, sino que es muy 
antigua. Las sociedades del pasado han utilizado para sus fines los vestigios materiales de culturas anteriores. En algún caso, como el del ejemplo, esta reutilización ha tenido un sentido ideológico. El obelisco, fabricado y ubicado originalmente en Egipto, fue trasladado a Roma en época imperial, como conmemora la inscripción que presenta su base. En época barroca, la plaza se decora con este milenario obelisco y se coloca una cruz en su parte superior como expresión del triunfo del cristianismo sobre la religión pagana.

Actividad 8. Base teórica sobre los procesos postdeposicionales de carácter geomorfológico

- Descripción: Explicación de los contenidos fundamentales sobre los procesos físico-mecánicos, edafo-químicos y catastróficos que afectan a los yacimientos arqueológicos, empleando para este fin una breve exposición oral apoyada en una presentación Powerpoint.

- Sentido formativo: Se imparten conceptos básicos sobre el tema referido. Estos conceptos y procesos constituyen una de las partes del tema que reviste mayor complejidad puesto que se relacionan con otras ciencias como la geología, la geografía y la química. Por tanto, se antoja necesaria una explicación de estas dinámicas por parte del profesor.

Actividad 9. Debate y puesta en común de posturas acerca de los problemas derivados de la clasificación funcional de los yacimientos

- Descripción: La clase comienza con una breve explicación por parte del profesor de los diferentes tipos de yacimientos arqueológicos que existen: lugares de habitación, lugares productivos, lugares rituales y lugares funerarios. A continuación, los alumnos deben responder a tres preguntas: ¿cuáles son las características generales que comparten todos estos 
tipos de yacimientos?, ¿todos los periodos históricos admiten esta división tipológica?, ¿conoces algún yacimiento que pueda incluirse en dos categorías tipológicas? A continuación, se genera un debate entre los representantes de los grupos, cada uno de ellos debe argumentar su respuesta y elegir, de nuevo por consenso, cuál es la repuesta más fundamentada. La actividad finaliza con la aclaración de conceptos por parte del profesor.

- Sentido formativo: Esta actividad persigue que los alumnos conozcan los rasgos básicos de cada uno de los diferentes tipos de yacimientos que se han propuesto. Al margen de esto, se busca que los estudiantes descubran por sí mismos que las categorías propuestas no funcionan como departamentos estancos, ya que existen yacimientos que se pueden incluir dentro de dos o más tipos. Esto último enlaza con el carácter relativo de las clasificaciones de yacimientos, aspecto sobre el que es muy necesario incidir.

\section{Actividad 10. Cuestionario final}

- Descripción: Al final de la clase, se entregó un cuestionario que los estudiantes realizaron también el primer día. Estas preguntas son leídas y contextualizadas por mí, sin condicionar o guiar, en ningún caso, las respuestas.

- Sentido formativo: Con esta actividad se pretende calibrar si las actividades realizadas en el ciclo de mejora han sido útiles y efectivas, en definitiva, si los alumnos han aprendido a través de estas herramientas docentes. 


\section{Aplicación del Ciclo de Mejora en el Aula}

\section{Relato resumido de las sesiones}

Actividad 1. Selección de una definición precisa de yacimiento arqueológico + Cuestionario inicial

Resultado y experiencia: En primer lugar, indiqué que los alumnos definieran con sus propios medios qué era para ellos un yacimiento arqueológico. Curiosamente, muchos alumnos levantaron la mano y fueron ofreciendo sus respuestas. Algunas de ellas incorporaban matices interesantes. La actividad finalizó con la elección por parte de los alumnos de la definición más precisa y completa de yacimiento arqueológico. En este punto, pude notar que la atención de los alumnos se difuminaba un poco, debido, seguramente, a la complejidad de alguno de los conceptos propuesto por los especialistas en la materia. A continuación, entregué el cuestionario inicial, creí oportuno no hacer ninguna aclaración ni guiar la resolución de las preguntas.

Actividad 2. Base teórica sobre los elementos que componen el registro arqueológico

Resultado y experiencia: En esta lección prioricé qué cuestiones eran más importantes y cuáles secundarias. La idea era introducir ciertos conceptos que se verían más tarde en otros temas. Sin embargo, la temática era profusa y muy compleja. Hay que tener en cuenta que para la mayoría de los alumnos es la primera asignatura del campo de la arqueología que cursan. Así pues, a la hora de definir y aclarar qué eran las unidades estratigráficas empleé más tiempo del que tenía pensado; esto hizo que la distribución horaria diseñada de antemano se descuadrara. 
Actividad 3. Debate y puesta en común de posturas acerca de las características fundamentales del registro arqueológico

Resultado y experiencia: Los alumnos respondieron bastante bien a esta actividad. Pudieron captar por sus propios medios que el registro arqueológico no nos devuelve todo lo que existió originalmente en el yacimiento. Los elementos de origen orgánico se degradan y, excepto circunstancias especiales, no pueden ser documentados por el arqueólogo. Los estudiantes reflexionaron acerca de que el registro arqueológico es parcial y que existen muchos artefactos y ecofactos que han desaparecido de él, ofreciéndonos, por tanto, una visión sesgada del pasado. En cuanto al debate, pude comprobar que era monopolizado por los alumnos más interesados en la materia, mientras que otros parecían desengancharse de la actividad.

Actividad 4. Aprendiendo a distinguir entre registro arqueológico y yacimiento arqueológico

Resultado y experiencia: La aclaración de conceptos con la que arrancó la clase se alargó un poco más de lo fijado inicialmente. De nuevo, estábamos ante conceptos complejos sobre los que había que construir el razonamiento posterior, de ahí que hiciera hincapié en que nadie se quedara sin preguntar en caso de que alguno tuviera dudas. A continuación, los estudiantes leyeron los artículos periodísticos, en algunos casos buscaban datos en el texto que no eran importantes para contestar a las preguntas. Les indiqué que lo esencial era el contexto de hallazgo y su conexión con una población o yacimiento de la época. La pregunta final era, quizás, demasiado ambiciosa y muchos alumnos no supieron contestar a la misma con certeza. Esto me sirvió para indicar cuál era la solución idónea y reforzar los conceptos que justificaban la realización de la actividad.

Actividad 5. Base teórica sobre los procesos que dan lugar a la formación del registro arqueológico 
Resultado y experiencia: La actividad transcurrió sin novedades; quizás debería haber sido más selectivo con la materia que quería explicar, nuevamente tardé más tiempo del establecido en desarrollar esta lección. Los alumnos parecían haber captado la explicación e hicieron preguntas al finalizar mi exposición.

Actividad 6. Debate y puesta en común de posturas acerca de las características de los yacimientos funerarios

Resultado y experiencia: De entrada, esta actividad parece gustar a los estudiantes. Una vez finalizado el visionado de los vídeos, éstos hicieron un esfuerzo importante para construir por grupos los rasgos de los yacimientos funerarios independientemente de su adscripción cultural o cronológica. Hubo cierto espíritu de competición por imponer a los demás grupos su visión del asunto y ofrecer unas respuestas fundamentadas. Para evitar que siempre hablaran los alumnos más aventajados propuse que cada respuesta fuera formulada por un miembro distinto del grupo. La actividad finalizó en el plazo propuesto y dejó un buen sabor de boca a profesor y alumnos.

Actividad 7. Alteración del registro arqueológico: expolio y reutilizaciones

Resultado y experiencia: Los alumnos supieron captar el sentido de la actividad. La gran mayoría razonó que la reutilización es una actividad consustancial al hombre, mientras que el expolio tiene otro sesgo más relacionado con la búsqueda ilegal de piezas arqueológicas. Se desató un debate interesante acerca del uso y tenencia de detectores de metales, incluso muchos alumnos me hicieron preguntas acerca del marco legal en que se inscribe la utilización de detectores. Este debate tuvo que ser reconducido y pregunté si los alumnos conocían algún caso de expolio con fines ideológicos. Me sorprendió la abundancia de respuestas.

Jornadas de Formación e Innovación Docente del Profesorado | № 2 (2019) Esta obra se distribuye con la licencia Creative Commons Reconocimiento-NoComercial-SinObraDerivada Internacional (CC BY-NC-ND 4.0.) 
Actividad 8. Base teórica sobre los procesos postdeposicionales de carácter geomorfológico

Resultado y experiencia: La explicación de esta base teórica se desarrolló con total normalidad. Esta vez sí pude adaptar la actividad al tiempo predeterminado que tenía. Algunos alumnos plantearon sus dudas, esto me sirvió para reforzar la explicación de algunos conceptos que, dada su complejidad, podrían haber sido más dificil de entender.

Actividad 9. Debate y puesta en común de posturas acerca de los problemas derivados de la clasificación funcional de los yacimientos

Resultado y experiencia: La explicación introductoria fue muy breve y solo esbocé alguna de las características de los yacimientos, puesto que los alumnos deberían responder a esta cuestión. La resolución de las preguntas llevó algún tiempo de más; de nuevo, los alumnos más diligentes solventaron estas preguntas rápidamente, mientras que otros tardaron bastante tiempo en hacerlo. La resolución de la tercera pregunta (¿cuántos tipos de yacimientos arqueológicos conoces?, ¿cuál es el fundamento de esta clasificación?) generó muchas dudas, tuve que contextualizarles la pregunta y guiar un poco la respuesta. El debate resultó menos dinámico de lo que yo esperaba. La actividad se resolvió en el tiempo estimado.

\section{Actividad 10. Cuestionario final}

Resultado y experiencia: En los últimos minutos de la clase se entregó el cuestionario que ya había pasado al principio del tema. Les recordé que este cuestionario sería anónimo y no tendría reflejo en su evaluación; no obstante, les indiqué que era muy importante, puesto que con él tendría que tasar el grado de efectividad de este ciclo de mejora. El tiempo dedicado a la resolución del cuestionario fue, quizás, escaso. Algunos alumnos se afanaban en ofrecer una respuesta completa a cada pregunta, mientras que otros redactaron una respuesta más concisa. 


\section{Evaluación del aprendizaje de los estudiantes}

El proceso de evaluación se ha realizado a los 20 alumnos matriculados en la asignatura.

Las preguntas del cuestionario (inicial y final) utilizado para dicho proceso de evaluación son las siguientes:

a) Define qué es un yacimiento arqueológico

b) ¿Qué diferencias existen entre un yacimiento histórico y un yacimiento arqueológico? Indica algún ejemplo de cada categoría.

c) ¿Cuántos tipos de yacimientos arqueológicos conoces?, ¿cuál es el fundamento de esta clasificación?

d) ¿Crees que el método arqueológico permite reconstruir todas las actividades humanas que han tenido lugar en el pasado?

e) ¿Qué actividades humanas o naturales pueden alterar un yacimiento arqueológico?, ¿qué consecuencias puede tener la perturbación del registro arqueológico?

Hemos elaborado una escalera de aprendizaje para situar los distintos niveles de respuestas a cada una de las preguntas. Estos niveles se fundamentan en una secuencia genérica progresiva de respuestas:

1. No sabe, no contesta o la respuesta no tiene relación alguna con la pregunta.

2. Desarrolla alguna idea básica sobre la cuestión.

3. Expresa suficiente conocimiento sobre el tema.

4. Ofrece una respuesta correcta, completa y fundamentada sobre la materia.

Los resultados de ambos cuestionarios se muestran en una gráfica en la que está representada cada una de las preguntas (Figura 4). La diferencia entre el cuestionario inicial y final muestra una evolución favorable del aprendizaje. 


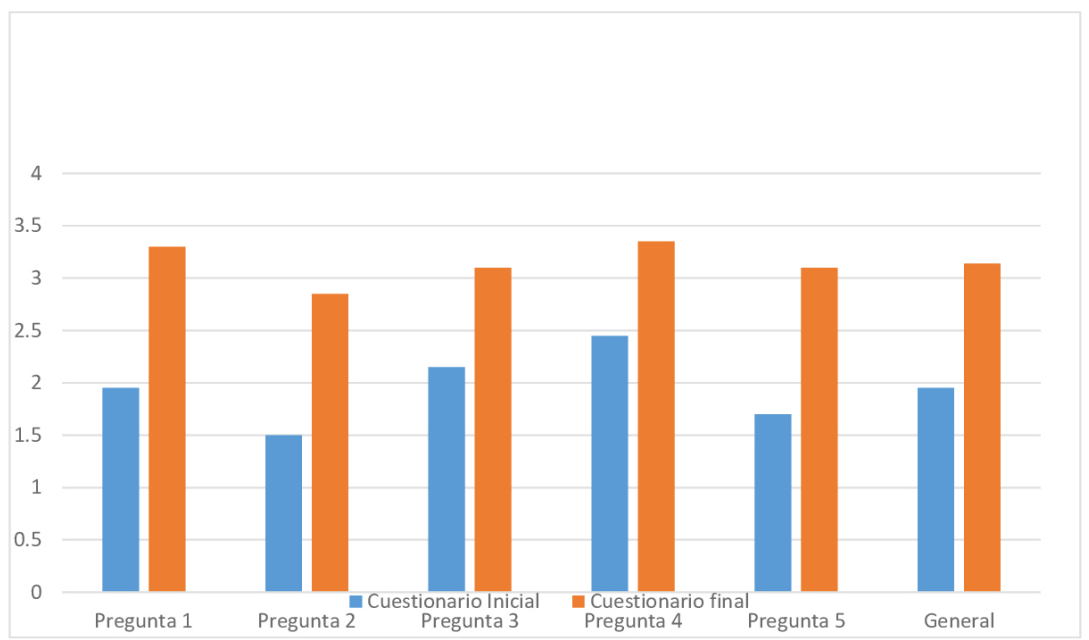

Figura 4: Medias de las respuestas a las cuestiones de los cuestionarios inicial y final.

Es interesante centrarnos en el comentario de la gráfica general, que recoge una media de los cuestionarios divididos por preguntas. En ella se observa una evolución bastante positiva con respecto a la situación inicial, de lo que se desprende que los métodos y técnicas puestos en práctica en este ciclo de mejora han sido eficaces y los alumnos han interiorizado los contenidos conceptuales y procedimentales de la unidad temática.

\section{Conclusiones finales}

El principio didáctico básico que he seguido en esta experiencia de innovación docente ha sido el aprendizaje basado en la construcción del conocimiento (Morales y Landa, 2004; Prieto, 2006). El trabajo del profesor, en este caso, consiste en fomentar la curiosidad y proporcionar el material idóneo para que los alumnos produzcan por sí mismos el saber (Bain, 2004). Este modelo ha parecido gustar a la mayor parte del alumnado y, como se ha podido comprobar, ha sido efectivo. Con todo, tiene también sus problemas; he percibido que los alumnos más 
preparados son los que participan más con este método y, por tanto, son los que más se benefician de él. Por el contrario, un porcentaje reducido de estudiantes parece ir a remolque del resto de la clase y muestra un escaso interés por las actividades propuestas.

El balance general de este CIMA es positivo, creo que los alumnos han asimilado este modelo de enseñanza. Por su parte, han colaborado en las actividades propuestas y demuestran su satisfacción, especialmente con el hecho de que ellos mismo vayan construyendo su conocimiento. Valoran especialmente que las clases sean más dinámicas y "entretenidas" comparadas con la típica lección expositiva. Un aspecto que ha resultado muy positivo es el fomento de la competencia entre los alumnos, este espíritu de competición les ha llevado a ofrecer lo mejor de cada uno. Eso sí, los estudiantes más motivados o preparados, en ocasiones, han monopolizado el discurso, como ya he señalado. En general, han tenido que trabajar en grupo, llegar a acuerdos entre ellos, aplicar la lógica y ofrecer respuestas fundamentadas en los conceptos que se han visto en clase. Otro aspecto a resaltar es que han ejercitado la oratoria, logrando vencer la timidez inicial que mostraban al principio de curso.

Finalmente, exponemos los principios didácticos (García Pérez y Porlán, 2017) que hemos puesto en práctica y que por su validez merecen ser aplicados en los cursos venideros:

- El profesor no debe ofrecer el saber como un producto acabado y perfecto, sino actuar como guía en el proceso de generación del conocimiento.

- Es básico desarrollar la colaboración entre docente y discentes para construir el conocimiento entre ambas partes.

- La elaboración de mapas conceptuales supone una herramienta bastante útil y clarificadora para estructurar la materia a impartir. 
- La formulación de situaciones o problemas cotidianos a los que deberán enfrentarse los alumnos en el desarrollo futuro de su profesión sirve para estimular el aprendizaje y mostrar la aplicación de los conceptos que se enseñan en las aulas.

- Es necesario crear en clase un clima de curiosidad científica y crítica constructiva enfocada al conocimiento.

- El empleo de nuevas tecnologías en el aula supone un estímulo para los alumnos y fomenta su participación en clase.

- El uso de cuestionarios constituye un instrumento para conocer el nivel inicial y el progreso de los estudiantes; además, permite identificar dificultades de aprendizaje.

Jornadas de Formación e Innovación Docente del Profesorado | № 2 (2019) Esta obra se distribuye con la licencia Creative Commons 


\section{Referencias bibliográficas}

Bain, K. (2007). Lo que hacen los mejores profesores universitarios. Valencia: Publicacions de la Universitat de València.

Finkel, D. (2008). Dar clase con la boca cerrada. Valencia: Publicacions de la Universitat de València.

García Pérez, F. F. y Porlán, R. (2017). Los Principios Didácticos y el Modelo Didáctico Personal. En R. Porlán (Coord.), Enseñanza Universitaria. Cómo mejorarla (pp. 93-104). Madrid: Morata.

Morales, P. y Landa, V. (2004): Aprendizaje basado en problemas. Theoria, 13, 145-157.

Prieto, L. (2006). Aprendizaje activo en el aula universitaria: el caso del aprendizaje basado en problemas. Miscelánea Comillas, 64(124), 173-196.

Rivero, A. y Porlán, R. (2017). La evaluación de la enseñanza universitaria. En R. Porlán (Coord.), Enseñanza Universitaria. Cómo mejorarla (pp. 73-92). Madrid: Morata.

Jornadas de Formación e Innovación Docente del Profesorado | № 2 (2019) Esta obra se distribuye con la licencia Creative Commons Reconocimiento-NoComercial-SinObraDerivada Internacional (CC BY-NC-ND 4.0.) 